\title{
Uncertainty justification of neutron cross section standards
}

\author{
Evgeny Gai and Vladimir Pronyaev \\ Institute of Physics and Power Engineering, Bondarenko sq. 1, 249033 Obninsk, Kaluga reg., Russia
}

\begin{abstract}
The existence of general measures for the covariance matrices of the evaluated data uncertainties obtained in different model and non-model least-squares fits of the same experimental data is considered. It is shown that some quantities (called measures here) calculated from the elements of covariance matrices are exactly and others are approximately conserved quantities independent from the model used in the fit. The use of these measures allows a simple comparison of uncertainties obtained in different evaluations of the same cross sections. The measures were used for the justification of the uncertainties of the neutron cross section standards evaluated in least-squares fits of a bulk volume of the experimental data. It is shown that the uncertainties obtained in the standards evaluation are realistic and determined completely by the uncertainties of the experimental data when taking into account all known correlations between the different experiments.
\end{abstract}

\section{Introduction}

Percentage errors, which are easy to compare, present important but not complete information about the uncertainty of the data. The complete data uncertainty is given by the covariance matrix of uncertainties, which generally has non-zero offdiagonal elements. These elements appear in the presentation of the uncertainties of the experimental data when the error propagation law is used for data reduction in the presence of common (for all points) components of the uncertainties (such as uncertainties in determination of the sample mass, neutron flux, etc.). A parametric model least-squares fit of the experimental data introduces additional intrinsic cross-energy correlations through the sensitivity coefficients of the cross sections relative to the model parameters.

Different model and non-model fits can be used in the evaluations of the same experimental data, and even if cross sections evaluated in different fits are close, the covariance matrices of the evaluated uncertainties can be very different. Usually the strong reduction of variances (or percentage uncertainties) is observed in the model fits comparing with the non-model fits. This effect, called often as a strong uncertainty reduction in the model fits, leads to cases, when evaluated uncertainties are considered by the experts, as unrealistic low. At the same time the uncertainty of some integral parameters (e.g., spectrum averaged cross sections) calculated with different covariance matrices can be very close. This is an indication that some general measures of uncertainty may exist, which can be used for comparison of uncertainties of evaluated data obtained in different model and non-model fits.

\section{Strict and approximate relations for covariance matrices of uncertainties}

Let us consider the parametric (model) least-squares fit of a single experimental data set in Bayesian approach with a prior having large uncertainties (non-informative prior). Because a prior in this case does not influence the results of the evaluation, the approach is fully equivalent to the non-Bayesian least-squares fit. The generalization for a few experimental data sets is simple: at first step - Bayesian non-model leastsquares fit with consecutive inclusion of all experimental data sets, and at second step - model least-squares fit of the result of the non-model least-squares evaluation. The final model evaluation should be the same as in the case of Bayesian model least-squares fit with consecutive addition of the experimental data sets. Because of this we will limit here our consideration to the case of the model least-squares fit of a single experimental data set.

If $\mathbf{R}$ is a covariance matrix of the uncertainties of experimental data, $\mathbf{G}$ is a matrix of sensitivity coefficients (partial derivatives of the model function with regard to the parameters) and superscripts $\mathrm{T}$ and -1 means transposition and inversion of the matrices, then the least-squares method gives for covariance matrix of the uncertainties of the evaluated parameters $\mathbf{W}$ :

$$
\mathbf{W}=\left(\mathbf{G}^{\mathrm{T}} \mathbf{R}^{-1} \mathbf{G}\right)^{-1}
$$

The covariance matrix of the evaluated uncertainties $\mathbf{V}$ given in the nodes where experimental data are presented can be written as:

$$
\mathbf{V}=\mathbf{G}^{\mathrm{T}} \mathbf{W G} \text {. }
$$

Using (1), (2) it is possible to show that the trace $(S p)$ of the matrices product $\mathbf{R}^{-1}$ and $\mathbf{V}$ does not depend on the model used, or quality of the fit assessed with $\chi^{2}$-criterion, but is equal to the number of the model parameters $M[1]$ :

$$
\begin{aligned}
S p \mathbf{R}^{-1} \mathbf{V} & =\sum_{i, k} R_{i, k}^{-1} V_{k, i}=\sum_{i, k} \sum_{\alpha, \beta} R_{i, k}^{-1} G_{\alpha, i}^{T} W_{\alpha, \beta}^{-1} G_{\beta, k} \\
& =\sum_{\alpha, \beta} W_{\alpha, \beta} W_{\alpha, \beta}^{-1}=M .
\end{aligned}
$$

The same is true for the matrices with the relative covariances $\mathbf{r}$ and $\mathbf{v}$.

$$
S p \mathbf{r}^{-1} \mathbf{v}=M
$$


Similarly, it can be shown, that:

$$
\begin{gathered}
\mathbf{V R} \mathbf{R}^{-1} \mathbf{V}=\mathbf{V} \\
\mathbf{v} \mathbf{r}^{-1} \mathbf{v}=\mathbf{v} .
\end{gathered}
$$

If the number of parameters is less than the number of data points, $\mathbf{V}$ and $\mathbf{v}$ have no inverse matrices, so $\mathbf{V} \neq \mathbf{R}$ and $\mathbf{v} \neq \mathbf{r}$. Relations (3)-(6) exhaust known to us relations strict for any model fit.

It can be shown that the next relations are strict for power polynomial fit in all cases and approximate, in general, for any other model fit of single experimental data set (or result of non-model least-squares fit for many data sets):

$$
\sum_{k, j} R_{i, k}^{-1} V_{k, j}=1,
$$

for any line $i$, and

$$
\begin{aligned}
& \sum_{i, k, j} R_{i, k}^{-1} V_{k, j}=N \\
& \operatorname{Det}(\mathbf{V}-\mathbf{R})=0
\end{aligned}
$$

where $N$ is the number of data points (energy nodes) and Det is the determinant of the matrix.

In case of power polynomial model, the quality of the fit does not influence the accuracy of the equalities (7)-(9), but it is not so in a general model case. The equalities were obtained analytically for a power polynomial model. In general case, they are based on conditions of necessity and sufficiency of existence of the solutions, when they are going through the experimental points. The equalities (3)-(9) were checked numerically in the fits with different Padé analytical expansion models.

The equalities (3)-(9) can be used for checks of covariance matrices of uncertainties of evaluated data obtained in different least-squares fits of the same experimental data.

Using the expression of the determinant through the matrix elements $\left(V_{i, k}-R_{i, k}\right)$ for the line $i$ and corresponding co-factors to these elements $A\left(V_{i, k}-R_{i, k}\right)$, we can write for any line $i=1, \ldots, N$ :

$$
\sum_{k} V_{i, k} A\left(V_{i, k}-R_{i, k}\right)=\sum_{k} R_{i, k} A\left(V_{i, k}-R_{i, k}\right)
$$

There are two general measures of uncertainty [1] that are strictly invariant for a wide range of model evaluations. Let $\bar{y}_{W G}$ is the mean value of the data $y\left(x_{k}\right)$, evaluated in points $x_{k}$ and weighted by the elements of the experimental covariance matrix:

$$
\bar{y}_{w a}=\frac{\sum_{i, k=1}^{N} R_{i . k}^{-1} y\left(x_{k}\right)}{\sum_{i, k=1}^{N} R_{i . k}^{-1}} .
$$

For all model evaluations with regression functions having a constant shift as one of the parameters, $y(x, p)=$ $p_{1}+g\left(x ; p_{2}, \ldots, p_{M}\right)$, the uncertainty of the weighted mean value of the evaluated data as defined by (11) does not

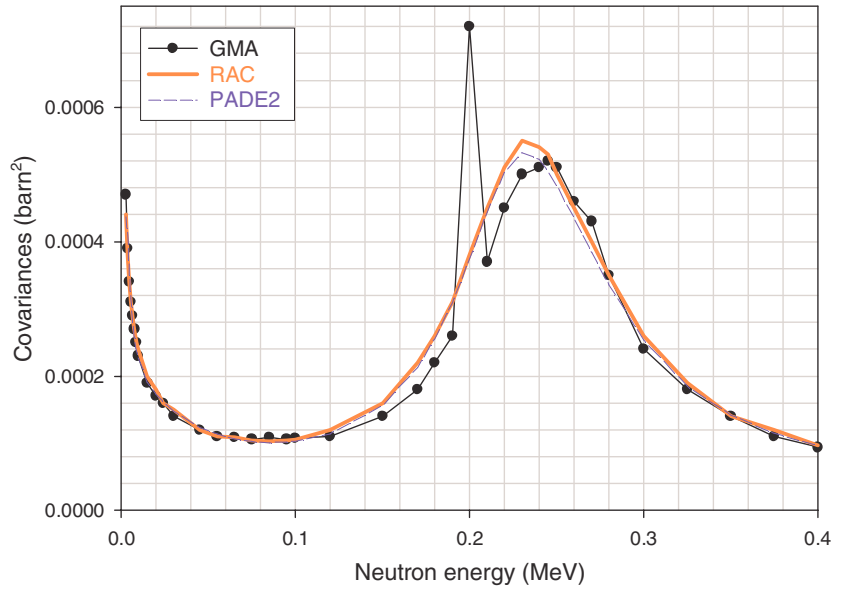

Fig. 1. Covariances between evaluated value at $0.2 \mathrm{MeV}$ and other points (line or row of the covariance matrix elements) obtained in the model (RAC R-matrix and PADE2 analytical expansion) and nonmodel (GMA) fits of ${ }^{6} \mathrm{Li}(\mathrm{n}, \mathrm{t})$ data.

depend on the model and is equal to the uncertainty of the weighted mean value of the experimental data:

$$
P_{w a}=\left\langle\left(\Delta \bar{y}_{w a}\right)^{2}\right\rangle=\frac{\sum_{i, k, l, m} R_{i, k}^{-1} V_{k, l} R_{l, m}^{-1}}{\left(\sum_{i, k} R_{i, k}^{-1}\right)^{2}}=\frac{1}{\left(\sum_{i, k} R_{i, k}^{-1}\right)} .
$$

In some cases, it is impossible to single out a constant shift as a parameter. If a constant factor can be singled out as one of the approximate parameters of the regression function, the relation similar to (12) can be written for the relative covariances. The relation for the relative covariances is also valid when (12) is valid for absolute covariances, but not vice versa.

Relation (10) shows that the sum of the elements of a line (row) of the covariance matrix of evaluated data weighted with their co-factors is close to the sum of the elements for the same line (row) of the covariance matrix of experimental data weighted with their co-factors. At the same time the practice of the fits using different model and non-model approaches had shown that even unweighted sums of the elements of the same line (row) of the evaluated data are very close when fits are close. To illustrate this, the comparison of the elements of the line (row) of covariance matrices of data evaluated for five experimental data sets of ${ }^{6} \mathrm{Li}(\mathrm{n}, \mathrm{t})$ standard cross section in non-model (GMA) and model (RAC R-matrix and PADE2 analytical expansion) [2] is shown on figure 1. The line (row) presents covariances between data at $0.2 \mathrm{MeV}$ neutron energy and data at other neutron energies. The value given at $0.2 \mathrm{MeV}$ presents the variance. As we see the variances are very different in the model and non-model fits, but the sum of the elements of the covariance matrix for given line (row) differs only at 1.6 per cent and this difference for the sum of all elements of covariance matrices evaluated in different approaches is even less. High value of variances in the nonmodel fit is compensated by higher values of covariances near diagonal of covariance matrix of uncertainties in the model fits. Because uncertainty of important integral quantities calculated with evaluated data may include different types 


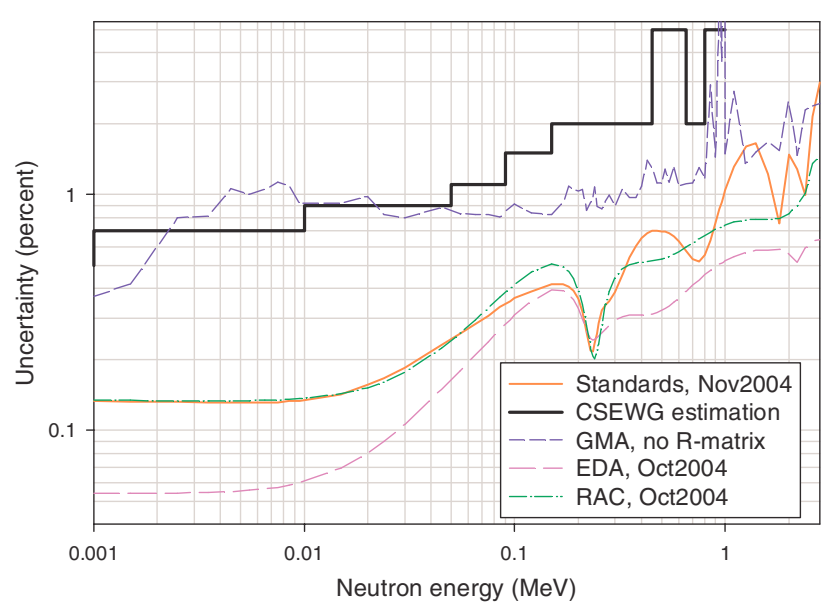

Fig. 2. Percentage uncertainties of the evaluated cross section for the ${ }^{6} \mathrm{Li}(\mathrm{n}, \mathrm{t})$ reaction: thick solid-line histogram - estimation given by CSEWG experts; short-dashed line - GMA result with full GMA database, including experimental data for the ${ }^{6} \mathrm{Li}(\mathrm{n}, \mathrm{t}),{ }^{6} \mathrm{Li}(\mathrm{n}, \mathrm{n})$ and ${ }^{6} \mathrm{Li}(\mathrm{n}$, total) reactions; thin solid line - uncertainties in the GMA combined fit for standards; long-dashed and dot-dashed lines - EDA and RAC R-matrix results, respectively.

of weighted sums, this property of least-squares fit is very important. Even if the covariance matrices of the uncertainties of the evaluated data look differently, uncertainty of integral parameters calculated with these data can be very close.

\section{Uncertainty of the standard cross sections}

The uncertainties obtained in an earlier standards evaluation [3] were considered to be unrealistically low by the experts of the US Cross Section Evaluation Working Group (CSEWG). New standards evaluation has the variances (or percentage uncertainties) close to the previous evaluation $[2,4]$. Figure 2 shows the percentage uncertainties for ${ }^{6} \mathrm{Li}(\mathrm{n}, \mathrm{t})$ standard cross section, where initial evaluation was obtained in the R-matrix model fit, and figure $3-$ for ${ }^{235} \mathrm{U}(\mathrm{n}, \mathrm{f})$, where initial evaluation was obtained with non-model GMA fit of the experimental data. The final evaluations were obtained as combined fit of R-matrix results for light nuclides with all available experimental data for heavy nuclides including ratios of the cross sections.

The CSEWG estimated the uncertainties as a possible corridor of errors within which 2/3-rd of modern days experimental results should fall. No estimation of correlative properties of the uncertainties is given. They did not account any reduction of the uncertainties because of inclusion of the relations between partial and total cross sections, use of experimental data for inverse channels of reactions and additional observables in the model fits. This is clearly seen from figure 2 for ${ }^{6} \mathrm{Li}(\mathrm{n}, \mathrm{t})$ reaction. Evaluated uncertainties in GMA non-model fit below $0.05 \mathrm{MeV}$ are very close to the CSEWG estimated uncertainties. Above $0.05 \mathrm{MeV}$ the measurements of total and elastic cross sections contribute in the reduction of the $(n, t)$ reaction uncertainty evaluated in non-model GMA fit. Use of R-matrix model substantially reduces the percentage uncertainties below $0.05 \mathrm{MeV}$ because

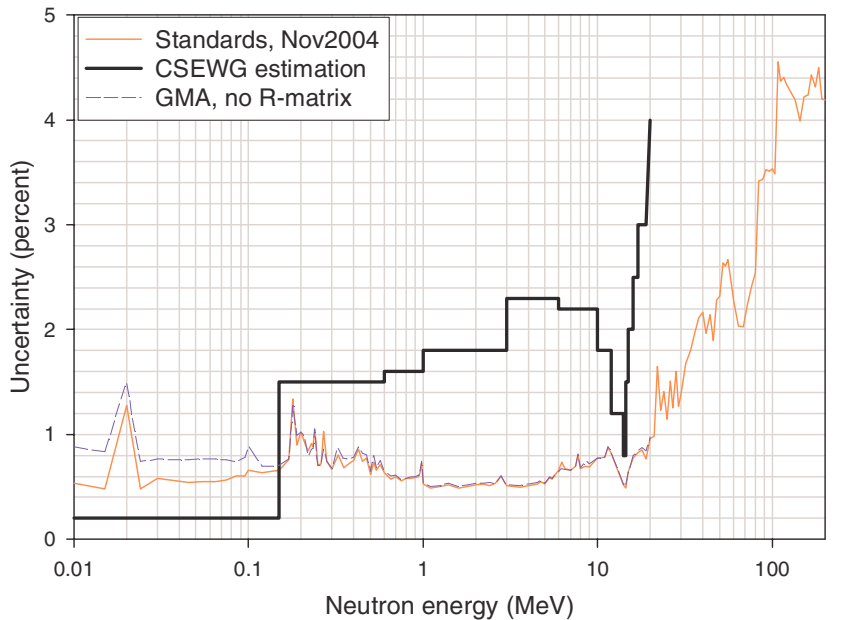

Fig. 3. Percentage uncertainties of the evaluated cross section for the ${ }^{235} U(n, f)$ reaction: thick solid-line histogram - estimation given by CSEWG experts; short-dashed line - GMA result with full GMA database, including experimental data for reactions induced by neutrons at ${ }^{6} \mathrm{Li}$ and ${ }^{10} \mathrm{~B}$; thin solid line - GMA combined fit for standards with inclusion of R-matrix results for ${ }^{6} \mathrm{Li}$ and ${ }^{10} \mathrm{~B}$.

of low uncertainty of the experimental data at thermal point propagates in the model fit to the range with $1 / v$ cross section behavior. The low percentage uncertainty is obtained also near the position of the resonance at $0.235 \mathrm{MeV}$. The difference between two R-matrix results is explained by the different presentation of the experimental uncertainties in EDA and RAC codes. Use in R-matrix fit a large amount of experimental data for inverse reaction channels and other observables, which are not accounted in GMA non-model fit, as well as common reduction of percentage uncertainties in the model comparing with non-model fits as shown in figure 1, explain the difference between CSEWG and ${ }^{6} \mathrm{Li}(\mathrm{n}, \mathrm{t})$ standard reaction evaluation uncertainty.

Influence of the R-matrix fit of ${ }^{6} \mathrm{Li}(\mathrm{n}, \mathrm{t})$ and ${ }^{10} \mathrm{~B}(\mathrm{n}, \alpha)$ reactions on the ${ }^{235} \mathrm{U}(\mathrm{n}, \mathrm{f})$ standard cross section is small and limited by the neutron energy below $0.2 \mathrm{MeV}$ (fig. 3). The low percentage uncertainties obtained from the GMA combined fit of the ${ }^{235} \mathrm{U}(\mathrm{n}, \mathrm{f})$ cross section for neutron energies between 0.5 and $10 \mathrm{MeV}$ are the consequences of low uncertainties (at 2 per cent level) assigned by the experimenters to the results of absolute cross section measurements. The same is true at $14 \mathrm{MeV}$, where no large differences between the GMA results and experts' estimation are observed. There are many high precision absolute measurements done with different methods at this point.

For analysis of uncertainties of the ${ }^{235} \mathrm{U}(\mathrm{n}, \mathrm{f})$ cross section in the energy range from 0.5 to $5 \mathrm{MeV}$ the spectral distribution of differences between the results of absolute measurements in this energy range and evaluated standard values were analyzed. The data were split up in 2 groups: measured before and after 1980. Measurements done after 1980 have been considered as modern measurements. We have clearly seen that the general spread of data is less for this group than for the group of old measurements. 2/3-rd of data points for modern experiments falls in less than $1.5 \%$ error corridor. This agrees with a CSEWG estimation in $1.5-2.3 \%$ for this energy 
range. Thorough analysis of uncertainties and account of all existing correlations was done for experimental data used in the evaluation of standards. The analysis included also search of outlaying data, which have substantially increased the chisquare value in the fit. It was observed for standard reactions that the data with large uncertainties are usually the outlaying data. Then the inclusion of the additional component of systematical uncertainty to these outlaying data making them consistent with a bulk of other data did not influence much the evaluated data and their uncertainties. As a consequence of lack of correlations between many measurements using different methods, samples, detectors, we see the reduction of the percentage uncertainty in least-squares fit to $0.5 \%$ for ${ }^{235} \mathrm{U}(\mathrm{n}, \mathrm{f})$ with rather high cross-energy correlations. The uncertainties evaluated in such approach can be considered as realistic because all factors that can lead to the increase of the evaluated uncertainties are accounted.

The pessimistic evaluation of the uncertainties is based on the least-squares fit in an approach similar to the approach used by the CSEWG experts for simple estimations. In this approach the statistical and systematical uncertainties assigned to the experimental data by the experimentalists were not taken into account, they were assigned using observable scatter (distribution) and shift of the data relative the model approximant in selected energy ranges. The use of this approach is possible when we have enough data for their statistical analysis. The analysis allows also estimate the off-diagonal covariances. Evaluated values obtained in PADE2 model fit of these data with covariance matrices of uncertainties estimated in such a way were rather close to the standards evaluation, the percentage uncertainties of the evaluated data were close to the values obtained with GMA non-model fit for ${ }^{6} \mathrm{Li}(\mathrm{n}, \mathrm{t})$ and ${ }^{235} \mathrm{U}(\mathrm{n}, \mathrm{f})$ reactions. But if we compare the average element of covariance matrices of the evaluated uncertainties, we will find the large differences: pessimistic evaluation gives 4 to 8 times higher values comparing with the realistic evaluation of the standards. It is interesting to compare the uncertainty of ${ }^{252} \mathrm{Cf}$ spectrum averaged cross sections for these two evaluations of uncertainties. For evaluated ${ }^{252} \mathrm{Cf}$ spontaneous fission neutron spectrum and covariance matrix of uncertainty as recommended by W. Mannhart [5] we will obtain the following uncertainty of the spectrum averaged cross sections: $1.29 \%$ for pessimistic and $0.518 \%$ for realistic ${ }^{6} \mathrm{Li}(\mathrm{n}, \mathrm{t})$ standard evaluation and $0.568 \%$ for pessimistic and $0.287 \%$ for realistic ${ }^{235} \mathrm{U}(\mathrm{n}, \mathrm{f})$ standard evaluation. The difference is fully explained by the difference in the assigning of the systematical uncertainty to the experimental data: through thorough analysis of all components of uncertainty for each data set using error propagation law as it was done in realistic evaluation of standards, or through analysis of the spread of the data without consideration of the uncertainties assigned by the authors as it was done in the pessimistic evaluation.

\section{Conclusion}

Comparison of the evaluated covariance matrices of the uncertainties obtained with realistic (present standards) and pessimistic estimation of experimental data errors shows once more that variances (or percentage uncertainties) cannot characterize alone the uncertainties of the evaluated data. The contribution of the off-diagonal covariances of the evaluated data uncertainty in the uncertainties of the integral parameters is very important. The average covariance matrix element is a general characteristic of the covariance matrix of uncertainties. The practice of the fits with different models of the same experimental data had shown that the average value of the element of the evaluated covariance matrix is an approximately conserving quantity. The following hypothesis based mainly at our empirical experience can be formulated: if different model and non-model fits of the same experimental data are close, the average elements of the evaluated covariance matrices in these fits are also close. Comparing with relations (3)-(5) this hypothesis is less justified, but it is rather convenient to use in the comparison of different covariance matrices. No large violations of this hypothesis were found during the tests done in the course of the evaluation of the standard cross sections in cases where covariance matrices of the experimental data are obtained with the use of the error propagation law.

\section{References}

1. E.V. Gai, in An International Evaluation of Neutron Cross Section Standards, Technical Series Report, IAEA, Vienna (to be published).

2. V.G. Pronyaev, S.A. Badikov, Chen Zhenpeng, A.D. Carlson, E.V. Gai, G.M. Hale, F.-J. Hambsch, H.M. Hofmann, N.M. Larson, D.L. Smith, Soo-Youl Oh, S. Tagesen, H. Vonach, in Proceedings of the International Conference on Nuclear Data for Science and Technology, Santa Fe, NM, USA, 26 Sept.-1 Oct. 2004, AIP Conference Proceedings 769, Part 1, 808 (2005).

3. H. Condé (ed.), Nuclear Data Standards for Nuclear Measurements, 1991 NEANDC/INDC Nuclear Standards File, Report NEANDC-311, NEA-OECD, Paris, France (1992).

4. A. Carlson, S. Badikov, Chen Zhenpeng, E. Gai, G. Hale, F.J. Hambsch, H. Hofmann, T. Kawano, N. Larson, SooYoul Oh, V. Pronyaev, D. Smith, S. Tagesen, H. Vonach (these proceedings).

5. W. Mannhart, in Proceedings of an Advisory Group Meeting on Properties of Neutron Sources. Leningrad, USSR, 9-13 June 1986, Report IAEA-TECDOC-410, IAEA, Vienna (1987), p. 158. 